\title{
Enhanced Efficacy and Reduced Hepatotoxicity by Combination of Gnaphalium affine Extract and Benzbromarone in the Treatment of Rats with Hyperuricemic Nephropathy
}

Fei Liu ${ }^{1,2 \#}$ Xi-Zi Liu ${ }^{1 \#}$ Qian Yang ${ }^{1}$ Shi-Yi Han ${ }^{1}$ Si-Yang Fan ${ }^{1 *}$

${ }^{1}$ State Key Lab. of New Drug and Pharmaceutical Process, Shanghai Institute of Pharmaceutical Industry, China State Institute of Pharmaceutical Industry, Shanghai, People's Republic of China

2 The Laboratory of Marine Glycodrug Research and Development, Marine Biomedical Research Institute of Qingdao, Qingdao, People's Republic of China
Address for correspondence Si-Yang Fan, PhD, State Key Lab. of New Drug and Pharmaceutical Process, Shanghai Institute of

Pharmaceutical Industry, China State Institute of Pharmaceutical Industry, 285 Gebaini Road, Shanghai 201203, People's Republic of China (e-mail: max_121@163.com).

Pharmaceut Fronts 2021;3:e129-e137.
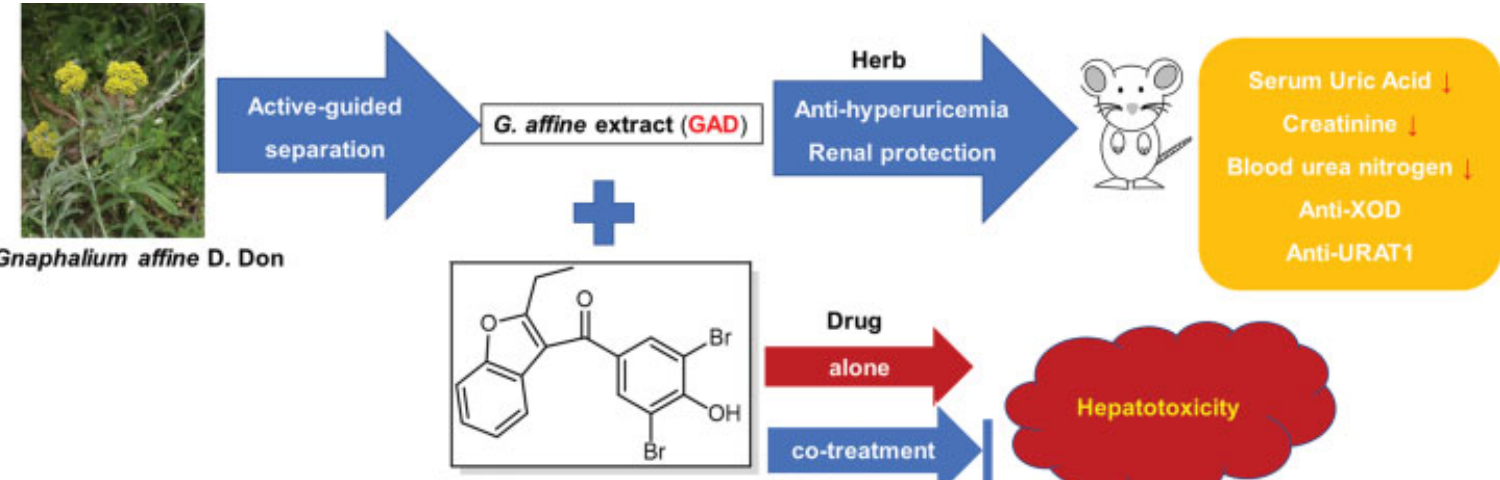

Gnaphalium affine D. Don
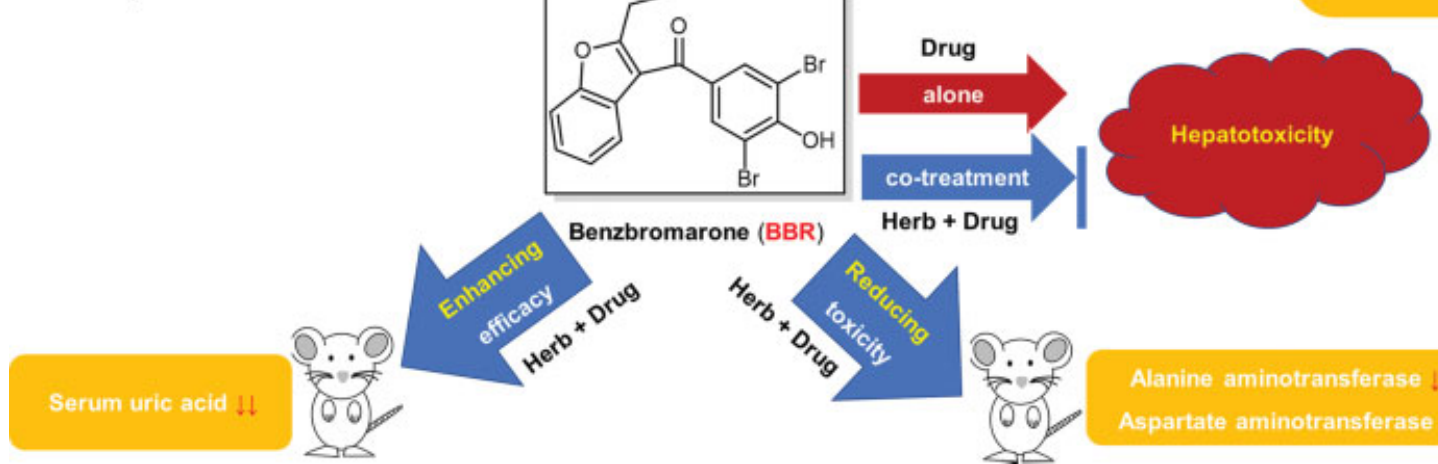

Herb + Drug
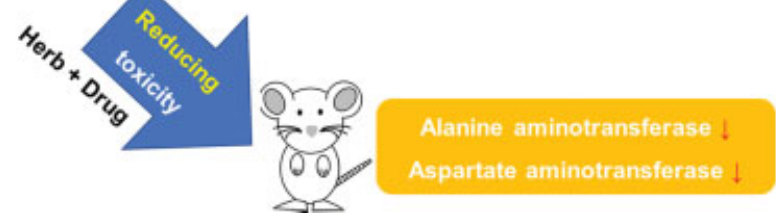

$\overline{\#}$ These authors contributed equally to this work.

received

July 21,2021

accepted

August 24, 2021
DOI https://doi.org/

$10.1055 / \mathrm{s}-0041-1736234$

ISSN 2628-5088. (c) 2021. The Author(s).

This is an open access article published by Thieme under the terms of the Creative Commons Attribution License, permitting unrestricted use, distribution, and reproduction so long as the original work is properly cited. (https://creativecommons.org/licenses/by/4.0/)

Georg Thieme Verlag KG, Rüdigerstraße 14, 70469 Stuttgart, Germany 


\author{
Abstract \\ Keywords \\ - Gnaphalium affine D. \\ Don \\ - benzbromarone \\ - co-treatment \\ - hyperuricemia \\ - hepatoprotection
}

Simultaneous oral intake of herbal medicine with chemical drugs may result in beneficial pharmacodynamic efficacy, including additive and synergistic effects with reduced toxicity. Gnaphalium affine D. Don (GAD) is a traditional Chinese Medicine that has been used for the management of hyperuricemia and gout. Benzbromarone (BBR) is one of the first-line drugs used for urate-lowering therapy in China but is toxic to the liver. The present study aimed to determine the effects of GAD and BBR, both alone and in co-treatment (with dosing interval of 1 hour), on chronic hyperuricemic nephropathy (HN) and hepatotoxicity in rats. Our data indicated that GAD significantly inhibited the elevation of serum uric acid, blood urea nitrogen, and creatinine levels in chronic $\mathrm{HN}$ rats at doses of 450 and $900 \mathrm{mg} / \mathrm{kg} /$ day. The rise in serum alanine aminotransferase and aspartate aminotransferase in BBR (or vehicle)-treated $\mathrm{HN}$ rats was significantly reduced by pre- (or post)-administration of GAD $(450 \mathrm{mg} / \mathrm{kg} /$ day). The $q$-value $>1.15$ (by jin method) indicated synergistic effects of co-treatments of BBR $(50 \mathrm{mg} / \mathrm{kg})$ with GAD $(450 \mathrm{mg} / \mathrm{kg})$. The synergistic beneficial effects were validated by comparison of BBR alone at a dose of clinical usage $(4.5 \mathrm{mg} / \mathrm{kg} /$ day, in two divided doses) and $\mathrm{BBR}+\mathrm{GAD}$ at half dose plus half dose $(2.25+225 \mathrm{mg} / \mathrm{kg} /$ day $)$ or half dose plus full dose $(2.25+450 \mathrm{mg} / \mathrm{kg} /$ day $)$. In conclusion, co-treatment with GAD and BBR holds promise for the management of hyperuricemia and gout.

\section{Introduction}

In China, due to the therapeutic benefits for enhancing efficacy and reducing side effects, the concomitant use of herbal medicine with conventional drug treatment plays a crucial role in the clinical therapies of major difficult and complicated diseases as well as major infectious diseases, such as cancers, ${ }^{1}$ rheumatoid arthritis, ${ }^{2}$ coronavirus diseases, ${ }^{3}$ etc.

Gnaphalium affine D. Don (family Compositae), commonly known as cudweed or Ching Ming vegetable (harvested around the Ching Ming festival and used to flavor the qingtuan, a traditional food of Chinese family), is a folk medicine used to treat rheumatic arthritis and gout. In previous studies, the ethanolic crude extract of the aerial parts of G. affine revealed promising hypouricemic effects. ${ }^{4,5}$ An activity-guided separation procedure was conducted to find the active fractions, which led to the findings of two fractions with different urate-lowering mechanisms (production inhibition and excretion promotion of urate) and the identification of active compounds (caffeoylquinic acids, flavonoids, and chalcone). A fraction also exhibited a nephroprotective effect against urate-induced renal dysfunction in mice. The two fractions were combined to obtain G. affine D. Don extract named "GAD." Other groups also reported that G. affine showed significant urate lowering effect on potassium oxonate (PO)-induced acute hyperuricemia and antigouty arthritis activity on monosodium urate (MSU) crystal-induced paw edema in mice. ${ }^{6}$ However, there are no reports about the hypouricemic and nephroprotective effects of G. affine in chronic hyperuricemic conditions. Moreover, G. affine (or G. tranzschelii) extract exhibited antioxidant activity against oxidative liver damage ${ }^{7}$ and a hepatoprotective effect on $\mathrm{CCl}_{4}$-induced liver jury in mice. ${ }^{8}$
Yet, there are no studies about the protective effect of $G$. affine on drug-induced hepatotoxicity.

As a uricosuric chemical agent, benzbromarone (BBR) is considered as the first-line drug for urate-lowering therapy at different stages of hyperuricemia and gout in China, ${ }^{9}$ in most other Asian countries, and also in some European countries. ${ }^{10}$ But it has not been approved for use in the United States and has recently been withdrawn from many European markets due to several reported cases of hepatotoxicity. ${ }^{10} \mathrm{~A}$ chronic toxicity study indicated that BBR exhibited little toxicity to healthy rat liver at a high dose of $50 \mathrm{mg} / \mathrm{kg}$ (10-fold more than the dosage for clinical use), ${ }^{11}$ but another study showed significant BBR-induced injury in obese mouse liver. ${ }^{12}$ Thus, BBR-induced hepatotoxicity may be associated with not only the drug but also the pathological situation.

We hypothesized that rats subjected to hyperuricemic nephropathy (HN) in combination with a chronic GAD medication may alleviate renal impairment and GAD and BBR cotreatment may show hepatoprotection with hypouricemia. The goal of this study was to determine the effects of GAD and BBR, both alone and in combination (co-treatment with a dosing interval of 1 hour), on chronic $\mathrm{HN}$ and hepatotoxicity. To the best of our knowledge, this is the first report on beneficial effects of combination therapy of BBR and G. affine.

\section{Materials and Methods}

\section{Reagents}

High-performance liquid chromatography (HPLC)-grade acetonitrile, formic acid, and methanol were obtained from Adamas- $\beta$ (Shanghai, China). Alanine aminotransferase (ALT) assay kit, aspartate aminotransferase (AST) assay kit, xanthine oxidase (XOD) activity assay kit, creatinine (CRE) assay kit, and blood urea nitrogen (BUN) assay kit were 
obtained from Nanjing Jiancheng Bioengineering Institute (Nanjing, China). Rat urate transporter 1 (URAT1) ELISA (enzyme-linked immunosorbent assay) kit was purchased from Shanghai Xitang Biotechnology Co., Ltd. (Shanghai, China). Uric acid (UA, Cas: 69-93-2), PO (Cas: 2207-75-2), adenine (Cas: 73-24-5), and BBR (Cas: 3562-84-3) were acquired from Sigma-Aldrich (St. Louis, United States), Macklin (Shanghai, China), Adamas- $\beta$ (Shanghai, China), and J\&K (Beijing, China), respectively. Sodium carboxymethylcellulose (CMC-Na) and other chemical reagents in analytical grade were purchased from Sinopharm Chemical Reagent Co., Ltd. (Shanghai, China).

The aerial parts of GAD were collected in Huangshanyi village, Jinhua, Zhejiang Province, China $\left(29^{\circ} 20^{\prime} 30^{\prime \prime} \mathrm{N}, 119^{\circ}\right.$ 54'25" E) in April 2018. Voucher specimens were identified by Prof. Tong $\mathrm{Wu}$ of Shanghai Institute of Pharmaceutical Industry and preserved at the Traditional Chinese Medicine Department of the institute (Herbarium No. 20180405).

\section{Preparation of GAD Extract}

Dried plant materials $(2.1 \mathrm{~kg}$ ) were extracted with $10 \mathrm{~L}$ of $60 \%$ $(\mathrm{v} / \mathrm{v})$ aqueous ethanol ( 2 hours) for four times. The combined ethanol extracts were evaporated in vacuum to obtain crude extract. The crude extract was suspended in water $(2.1 \mathrm{~L})$ and sequentially partitioned with petroleum ether $(2.1 \mathrm{~L} \times 3)$, EtOAc $(2.1 \mathrm{~L} \times 3)$, and $n-\mathrm{BuOH}(2.1 \mathrm{~L} \times 3)$, respectively. The EtOAc and $n$-BuOH fractions were combined and dried under vacuum at $60^{\circ} \mathrm{C}$ to obtain GAD.

\section{Animals}

Male Sprague-Dawley rats ( $180 \pm 10 \mathrm{~g}$ ) were purchased from Sino-British-SIPPR/BK Laboratory Animal Ltd (Shanghai, China). The animals were housed at the temperature of $20 \pm 2^{\circ} \mathrm{C}$, humidity of $50 \pm 5 \%$, and with 12 hours dark-light cycle for at least 1 week before the experiments. Distilled water and sterilized food for rats were available ad libitum. All animal experiments, which were conformed to the $\mathrm{Na}$ tional Institutes of Health Guidelines on Laboratory Research and Guide for the Care and Use of Laboratory Animals (Eighth Edition, 2011), were approved by the Animal Ethical Committee of Shanghai Institute of Pharmaceutical Industry.

\section{Study Design}

The study is composed of three experiments. In experiments 1,2 , and 3, GAD alone, GAD and BBR (toxic dose) in combination, or GAD and BBR (half a clinical dose) in combination were given, respectively.

Experiment 1: Antihyperuricemic activity was assayed using the HN rat model, which has been previously described in detail. ${ }^{13}$ The model was established and maintained by oral administration of a mixture of adenine $(0.1 \mathrm{~g} / \mathrm{kg})$ and PO $(1.5 \mathrm{~g} / \mathrm{kg})$ daily consistently for 28 days (once daily at 9:00 a.m.). Briefly, 40 rats were weighted and randomly divided into five groups: normal control (NC) group (0.5\% CMC-Na), HN group (0.5\% CMC-Na), HN treated with BBR $(10 \mathrm{mg} / \mathrm{kg} /$ day $)$ group, HN treated with GAD (450 mg/kg/day) group, and HN treated with GAD
(900 mg/kg/day) group by gavage (-Table 1). Treatment of BBR (suspended in 0.5\% CMC-Na) and GAD (suspended in $0.5 \%$ CMC-Na) started from day 3 to day 28 (the end of the experiment) once daily, 2 hours after administration of adenine and PO. On day 7, 14, 21, and 28, 2 hours after feeding of drugs (or vehicle), blood samples were taken and maintained at room temperature for 1 hour. The serum was obtained after centrifugation at 3,000 $\mathrm{g}$ for 10 minutes. The serum samples were stored at $-20^{\circ} \mathrm{C}$ until the measurement of UA (by HPLC method), CRE (by colorimetric method, diagnostic kit), and BUN (by colorimetric method, diagnostic kit) levels. At the end of the experiment (day 28), the rats were sacrificed after the last blood sampling according to ethical animal euthanasia guidelines. The kidneys and livers were collected immediately, washed in $0.9 \%$ saline, weighted, and rapidly stored at $-80^{\circ} \mathrm{C}$ until processing. Livers and kidneys were homogenized in $0.9 \%$ saline $(10 \%)$. The homogenate was centrifuged at $3,000 \mathrm{~g}$ for 10 minutes at $4^{\circ} \mathrm{C}$. The upper layers of kidney and liver were used for the assessment of URAT1 level (by ELISA method) and XOD activity (by colorimetric method) according to manufacturer's instruction of diagnostic kits.

Experiment 2: A hepatotoxic dose of $50 \mathrm{mg} / \mathrm{kg}$ was chosen based on a literature of BBR chronic toxicity test. ${ }^{11}$ Rats received adenine and $\mathrm{PO}$ daily consistently to keep $\mathrm{HN}$ for 28 days. Briefly, 48 male rats were weighted and randomly divided into six groups: NC group, HN group, HN treated with GAD (450 mg/kg/day) group, HN treated with GAD ( $450 \mathrm{mg} / \mathrm{kg} /$ day, 1 hour prior to BBR administration) and BBR ( $50 \mathrm{mg} / \mathrm{kg} /$ day) group, HN treated with BBR (50 $\mathrm{mg} / \mathrm{kg} /$ day) group, and HN treated with BBR $(50 \mathrm{mg} / \mathrm{kg} /$ day, 1 hour prior to GAD administration) and GAD (450 $\mathrm{mg} / \mathrm{kg} /$ day) group ( - Table 1). All drugs were given orally (po) from day 3 to day 28 (the end of the experiment), and the first dose of each day was given 1 hour after the administration of adenine and PO. On day 7, 14, 21, and 28,2 hours after the second dosing of the drug (or vehicle), blood samples were collected for the measurement of serum UA (by HPLC method), ALT (by colorimetric method, diagnostic kit), and AST (by colorimetric method, diagnostic kit) levels.

Experiment 3: The efficacy of BBR at a dose of clinical usage $(4.5 \mathrm{mg} / \mathrm{kg} /$ day, in two divided doses) was in comparison with that of $B B R+G A D$ at half dose plus half dose $(2.25+225 \mathrm{mg} / \mathrm{kg} /$ day $)$ or half dose plus full dose $(2.25+450 \mathrm{mg} / \mathrm{kg} /$ day $)$. Rats received adenine and PO daily consistently for 21 days. Briefly, 40 male rats were weighted and randomly divided into five groups: NC group, HN group, HN treated with BBR $(4.5 \mathrm{mg} / \mathrm{kg} /$ day with two divided doses at 1-hour intervals) group, HN treated with BBR $(2.25 \mathrm{mg} / \mathrm{kg} /$ day, 1 hour prior to GAD administration) and GAD (225 mg/kg/day) group, and HN treated with BBR $(2.25 \mathrm{mg} / \mathrm{kg} /$ day, 1 hour prior to GAD administration $)$ and GAD $(450 \mathrm{mg} / \mathrm{kg} /$ day $)$ group (-Table 1). All drugs were given orally (po) daily for 21 days, and the first dose of each day was given 1 hour 


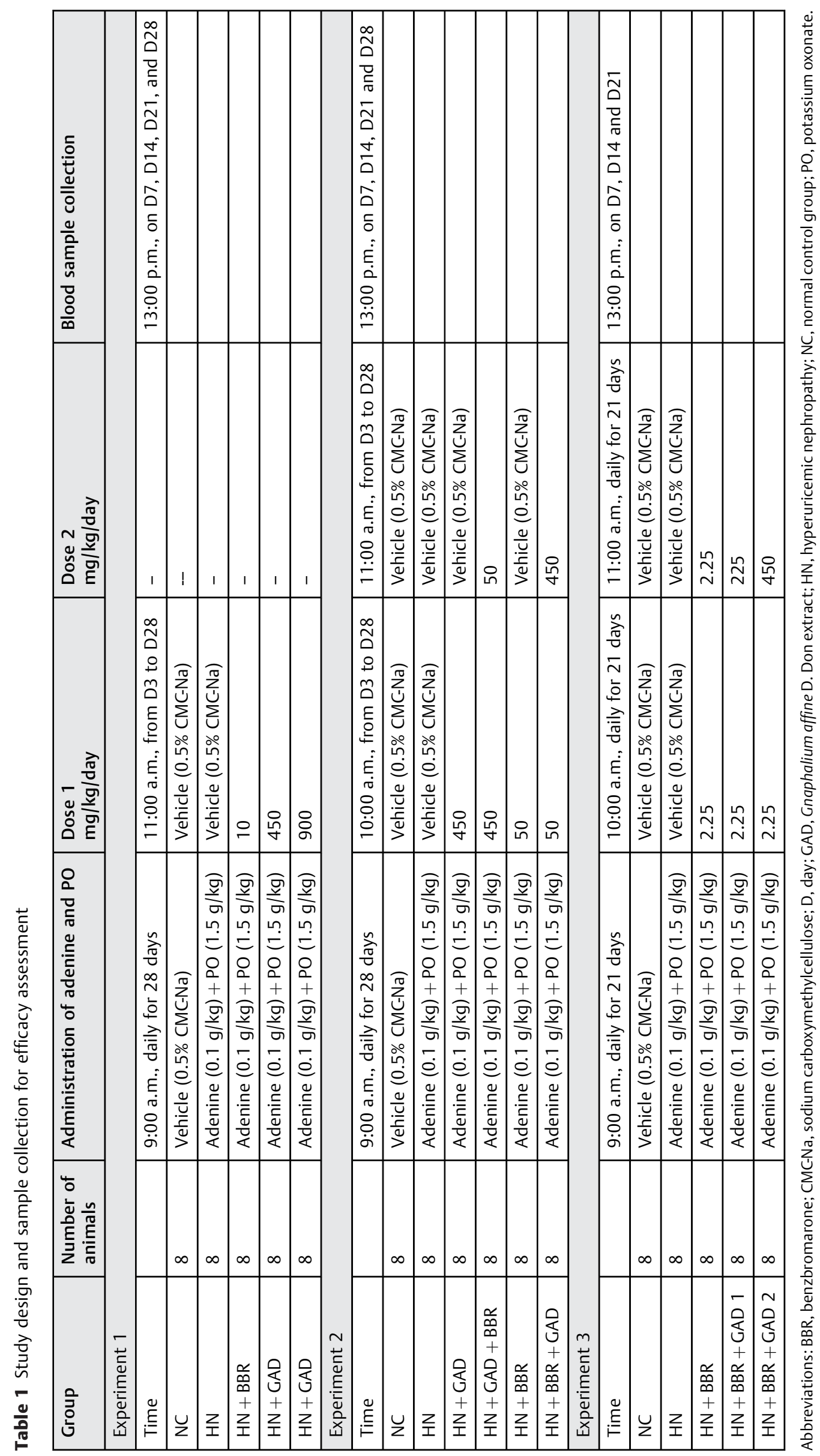

Pharmaceutical Fronts Vol. 3 No. 3/2021 $\quad$ @ 2021. The Author(s). 
Enhanced Efficacy and Reduced Hepatotoxicity of GAD + BBR in HN Rats Liu et al. e133

Table 2 Effect of GAD (450 and $900 \mathrm{mg} / \mathrm{kg}$, po) and BBR $(10 \mathrm{mg} / \mathrm{kg}$, po) on BW in HN rat model

\begin{tabular}{|c|c|c|c|c|c|}
\hline \multirow[t]{2}{*}{ Group } & \multicolumn{5}{|l|}{ BW (g) } \\
\hline & D0 & D7 & D14 & D21 & D28 \\
\hline NC & $175.4 \pm 32.9$ & $227.5 \pm 19.0$ & $258.6 \pm 16.0$ & $276.0 \pm 15.5$ & $306.4 \pm 17.0$ \\
\hline $\mathrm{HN}$ & $177.5 \pm 29.2$ & $211.8 \pm 21.7$ & $241.3 \pm 27.2$ & $246.0 \pm 19.1^{* *}$ & $264.0 \pm 27.9^{* *}$ \\
\hline $\mathrm{HN}+\mathrm{GAD}(450 \mathrm{mg} / \mathrm{kg})$ & $174.8 \pm 28.4$ & $199.6 \pm 18.6^{*}$ & $225.0 \pm 16.9^{* *}$ & $250.0 \pm 14.3^{* *}$ & $282.7 \pm 14.2^{*}$ \\
\hline $\mathrm{HN}+\mathrm{GAD}(900 \mathrm{mg} / \mathrm{kg})$ & $181.3 \pm 30.9$ & $203.9 \pm 26.1$ & $232.0 \pm 13.8^{* *}$ & $248.3 \pm 11.5^{* *}$ & $279.0 \pm 11.9^{* *}$ \\
\hline $\mathrm{HN}+\mathrm{BBR}(10 \mathrm{mg} / \mathrm{kg})$ & $173.0 \pm 21.8$ & $224.8 \pm 12.8$ & $237.4 \pm 16.7^{*}$ & $248.9 \pm 20.8^{*}$ & $278.4 \pm 17.9^{* *}$ \\
\hline
\end{tabular}

Abbreviations: BBR, benzbromarone; BW, body weight; D, Day; GAD, Gnaphalium affine D. Don extract; HN, hyperuricemic nephropathy; NC, normal control group; po, per os.

Note: Results are expressed as mean \pm SD $(n=8)$ and analyzed using one-way ANOVA followed by Dunnett's test. ${ }^{*} p<0.05,{ }^{* *} p<0.01$.

after the administration of adenine and PO. On day 7,14 , and 21, 2 hours after the second dosing of the drug (or vehicle), blood samples were collected for the measurement of serum UA level by HPLC method.

\section{UA Measurement}

UA level in serum was determined by the HPLC method detailed in the literature. ${ }^{14}$ Briefly, $50 \mu \mathrm{L}$ of the serum samples was vortex-mixed with $100 \mu \mathrm{L}$ of methanol for 1 minute. The samples were transferred for cold centrifugation $\left(4^{\circ} \mathrm{C}\right)$ at $14,000 \mathrm{~g}$ for 10 minutes. After centrifugation, $10 \mu \mathrm{L}$ of the upper layer was injected into a Thermo Scientific U3000 HPLC system, equipped with quaternary pump, an autosampler, a diode array detector, and Chromeleon software (Thermo Scientific, United States). Separation was conducted on a Zorbax SB-C18 column $(4.6 \times 250 \mathrm{~mm}, 5$ $\mu \mathrm{m})$ (Agilent Technologies, United States) at $30^{\circ} \mathrm{C}$ with the mobile phase: water ( $0.5 \%$ acetic acid) (A) and methanol (B) under the following conditions: 0 to 10 minutes $5 \% \mathrm{~B} ; 10$ to 20 minutes $100 \%$ B; 20 to 30 minutes $5 \%$ B. The flow rate was $1.0 \mathrm{~mL} / \mathrm{min}$ and the detection wavelength was $288 \mathrm{~nm}$.

\section{Statistical Analysis}

Data are expressed as the mean \pm standard deviation or the mean \pm standard error of the mean. Statistical analysis between groups was performed using one-way ANOVA (analysis of variance) followed by Dunnett's test. All results with $p<0.05$ were considered statistically significant.

\section{Preliminary Analysis of the Interaction between GAD and BBR}

The Jin method and Bürge method were used to evaluate the interaction between GAD and BBR, preliminarily. ${ }^{15}$ The Jin method provides a " $q$ "-value, according to which the interaction between two drugs can be classified as an antagonistic effect ( $q \leq 0.85$ ), additive effect $(0.85 \leq q<1.15)$, or synergistic effect $(q \geq 1.15)$. And the formula is $q=E_{\mathrm{a}+\mathrm{b}} /\left(E_{\mathrm{a}}+E_{\mathrm{b}}\right.$ $-E_{\mathrm{a}} \times E_{\mathrm{b}}$ ), where $E_{\mathrm{a}+\mathrm{b}}, E_{\mathrm{a}}$, and $E_{\mathrm{b}}$ are the effect (UA inhibition ratio) of combination treatment, effect (UA inhibition ratio) of drug A only, and effect (UA inhibition ratio) of drug B only, respectively. The Bürge method provides a " $q$ "-value, according to which the interaction between two drugs can be classified as an antagonistic effect $(q<1)$, an additive effect $(q=1)$, or a synergistic enhancing effect $(q>1)$. And the formula is $q=E_{(\mathrm{a} / 2+\mathrm{b} / 2)} / E_{\mathrm{a}}\left(\right.$ or $\left.E_{\mathrm{b}}\right)$, where $E_{(\mathrm{a} / 2+\mathrm{b} / 2)}, E_{\mathrm{a}}$, and $E_{\mathrm{b}}$ are the effect (UA inhibition ratio) of combination of two drugs at a half dose for each, effect (UA inhibition ratio) of drug A only at a full dose, and effect (UA inhibition ratio) of drug B only at a full dose, respectively.

\section{Results}

Effects of GAD on Hyperuricemia and Renal Function in HN rats (Results from Experiment 1)

Over 21 days, a significant decrease in body weight (BW) gain was observed in HN-group rats versus NC-group rats $(p<0.01)$ (-Table 2). There were significant increases in relative kidney weight (kidney-to-BW ratio) and relative liver weight (liver-to-BW ratio) in HN-group rats compared with NC-group rats $(p<0.01, p<0.001)$ ( - Fig. 1). Rats treated with GAD at $900 \mathrm{mg} / \mathrm{kg}$ showed significant decreases in relative kidney weight $(p<0.05)$ and relative liver weight $(p<0.05)$ when compared with the HN-group rats ( - Fig. 1). BBR at $10 \mathrm{mg} / \mathrm{kg}$ failed to significantly reduce the increase in relative kidney weight $(p>0.05)$ and relative liver weight $(p>0.05)$ when compared with the HN-group rats (-Fig. 1).

The effects of GAD and BBR on indices of hyperuricemia (serum UA) and renal function (serum CRE and BUN-the important markers of renal dysfunction ${ }^{16}$ ) are shown in - Fig. 1. HN rats showed a remarkable increase in serum UA $(p<0.001)$, CRE $(p<0.01)$, and BUN $(p<0.01)$ when compared with the $\mathrm{NC}$ rats from day 7 and the hyperuricemia and the renal damage progressively aggravated over time (-Fig. 1). GAD (450 and $900 \mathrm{mg} / \mathrm{kg}$ ) and BBR (10 mg/kg) treatment showed significant effects on urate levels (-Fig. 1A) in HN rats from day 21. GAD (450 and $900 \mathrm{mg} / \mathrm{kg}$ ) treatment showed significant inhibitions of BUN and CRE production from day 14 ( - Fig. 1B, C). BBR (10 mg/kg) had no significant effects on BUN and CRE levels in HN rats $(p>0.05$ vs. HN group) (-Fig. 1B, C). The liver XOD activity and the kidney URAT1 levels were significantly elevated in the HN group compared with the NC group $(p<0.001)$. GAD (450 and $900 \mathrm{mg} / \mathrm{kg})$ and BBR $(10 \mathrm{mg} / \mathrm{kg})$ caused a significant inhibition of liver XOD activity, especially GAD (450 and $900 \mathrm{mg} / \mathrm{kg}$ ), that caused an enzyme inhibition of 59.7 and 74.2\%, respectively (- Fig. 1D, E). GAD (450 and $900 \mathrm{mg} / \mathrm{kg}$ ) 
A

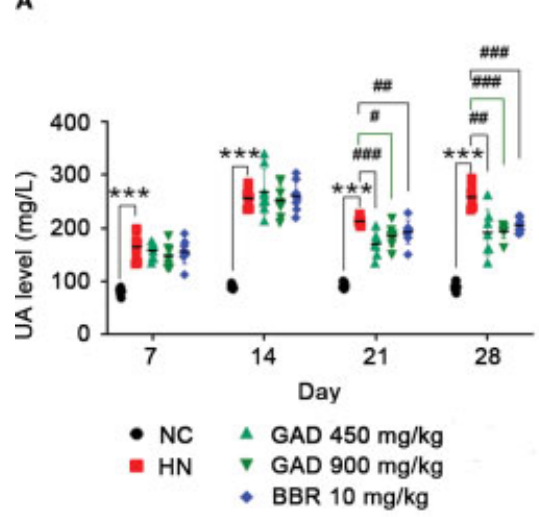

D

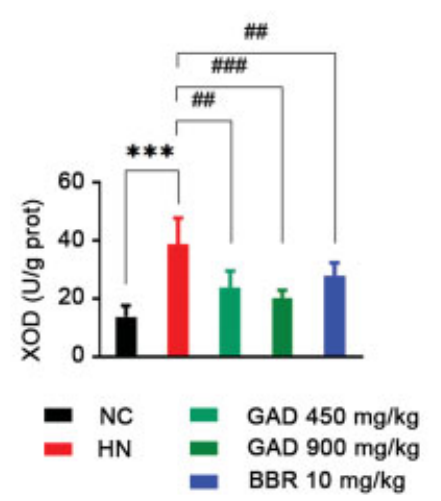

B

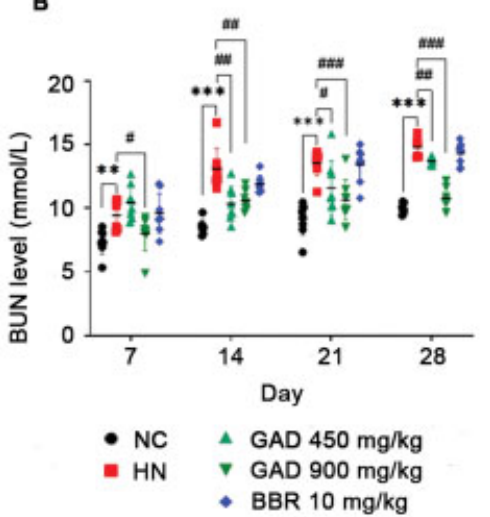

E

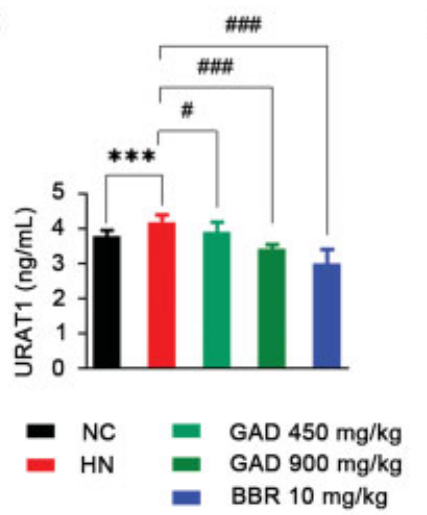

C

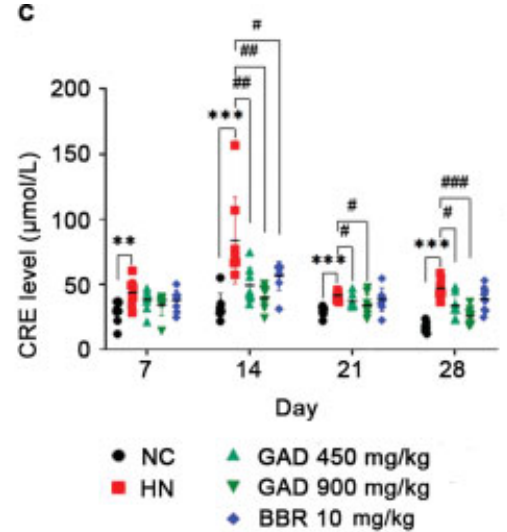

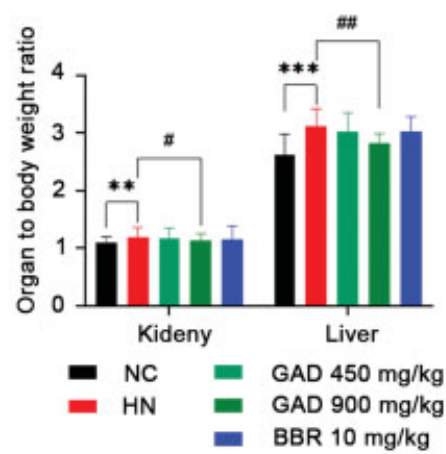

Fig. 1 Effects of GAD and BBR rats on (A) serum UA, (B) BUN level, (C) creatinine (CRE), (D) XOD activity, (E) kidney urate transporter 1 (URAT1) level, and (F) relative kidney weight (kidney-to-body weight ratio) and relative liver weight (liver-to-body weight ratio) in rats with HN. Values were expressed as mean \pm SD of eight animals. For statistical significance, one-way ANOVA was used followed by Dunnett's test. ${ }^{* *} p<0.01$, versus NC group; ${ }^{* * *} p<0.001$, versus NC group. ${ }^{\#} p<0.05$, versus HN group; ${ }^{\# \#} p<0.01$, versus $\mathrm{HN}$ group; ${ }^{\# \# \#} p<0.001$, versus HN group. BBR, benzbromarone; BUN, blood urea nitrogen; CRE, creatinine; GAD, Gnaphalium affine D. Don extract; HN, hyperuricemic nephropathy; NC, normal control; SD, standard deviation; UA, urate; XOD, liver xanthine oxidase.

and $\mathrm{BBR}(10 \mathrm{mg} / \mathrm{kg})$ treatment significantly reduced the kidney URAT1 levels compared with those of untreated HN rats $(p<0.05, p<0.001)$ ( - Fig. 1E).

\section{Antihyperuricemic and Hepatoprotective effects of GAD used in Co-treatment with BBR (Results from Experiments 2 and 3)}

The effects of GAD, BBR, or their combination on indices of hyperuricemia and liver function (serum ALT and AST) are shown in -Fig. 2. Compared with NC (vehicle-treated) values, oral administration of rats with adenine $(0.1 \mathrm{~g} / \mathrm{kg} / \mathrm{d}$ for 28 days) and PO (1.5 g/kg/d for 28 days) caused increases in serum ALT and AST levels, but with no statistical significance ( $p>0.05$, vs. NC group). The co-administration of adenine and PO and the high dose of BBR $(50 \mathrm{mg} / \mathrm{kg} /$ day for 26 days) caused significant increases in serum ALT $(p<0.001$, compared with NC group from day 28$)$ and AST $(p<0.05, p<0.01$, and $p<0.001$, compared with NC group from day 7) levels. BBR at a dose of $50 \mathrm{mg} / \mathrm{kg}$ aggravated hepatotoxicity in HN rats. The rises in serum ALT and AST seen in BBR (or vehicle)-treated HN rats were significantly reduced by pre- (or post)-administration of GAD $(450 \mathrm{mg} / \mathrm{kg} /$ day). As shown in - Fig. $\mathbf{2 A}$ and $\mathbf{B}$, the 26-day continuous treatment with GAD in $\mathrm{HN}$ rats almost completely abolished the increase in serum ALT and AST levels, which were similar with those in NC rats. Compared with BBR alone, co-treatment with GAD and BBR in HN rats significantly inhibited the increase in ALT level from day $28(p<0.01, p<0.001)$ and AST level from day 7 to the end of the experiment $(p<0.05$, $p<0.01$ ), and the posttreatment of GAD (BBR + GAD) was more effective in hepatoprotection.

-Fig. 2C shows the antihyperuricemic effects obtained with the chemical and herbal drugs administered either alone (BBR or GAD) or in different administration orders of co-treatment $(\mathrm{GAD}+\mathrm{BBR}$ or $\mathrm{BBR}+\mathrm{GAD})$. The maximal urate-lowering effects of the co-treatments of BBR and GAD were obtained on day 28 (after 26-day drug administration) with either $80.8 \%$ (GAD + BBR) or $97.7 \%(B B R+G A D)$ inhibition ratio, respectively. As can be seen in - Fig. 2C, GAD and BBR co-treatments on most blood sampling days produced antihyperuricemic effects higher in magnitude when compared with the corresponding effects produced by the BBR alone, but there were no significant statistical differences (BBR + GAD vs. BBR on day 7, $p=0.078$; BBR + GAD vs. BBR on day $21, p=0.066$ ). Surprisingly, $q$-value $>1.15$ (calculated by the Jin method) in - Fig. $\mathbf{2 C}$ indicated that the synergistic effects appeared for the co-treatments of BBR $(50 \mathrm{mg} / \mathrm{kg}$ ) with GAD $(450 \mathrm{mg} / \mathrm{kg})$ on day 7 . As the same to its 


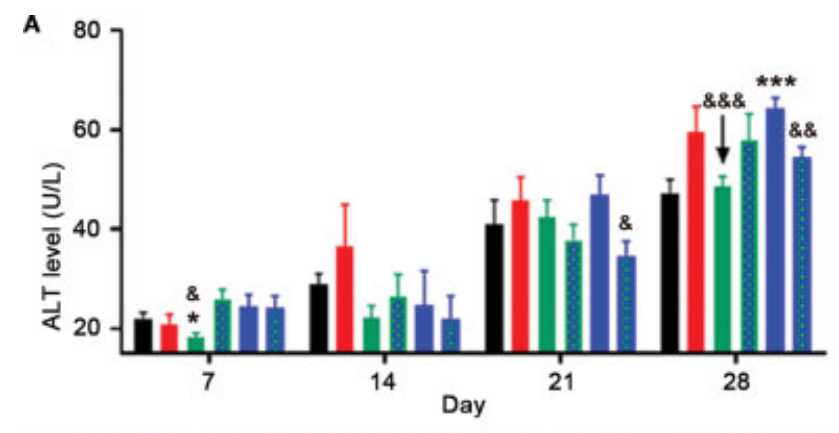

- $=\mathrm{NC}=\mathrm{HN}=\mathrm{EAD} 450 \mathrm{mg} / \mathrm{kg}=\mathrm{mAD} 450 \mathrm{mg} / \mathrm{kg}+\mathrm{BBR} 50 \mathrm{mg} / \mathrm{kg}$ - BBR $50 \mathrm{mg} / \mathrm{kg}=\mathrm{BBR} 50 \mathrm{mg} / \mathrm{kg}+\mathrm{GAD} 450 \mathrm{mg} / \mathrm{kg}$

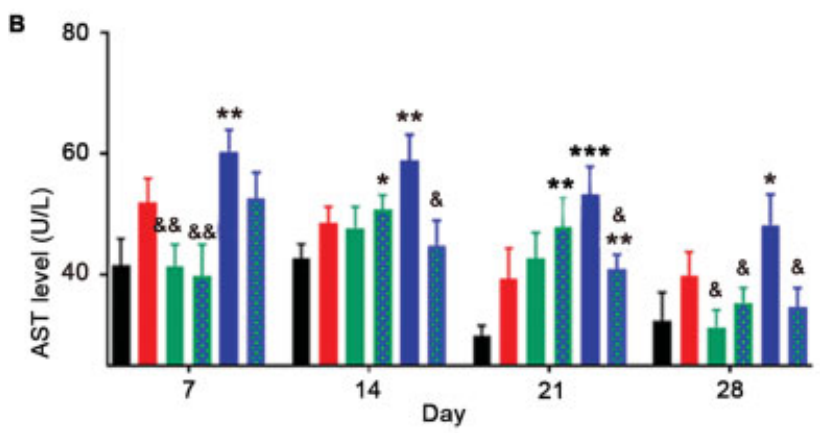

- $\mathrm{NC}=\mathrm{HN}=\mathrm{EAD} 450 \mathrm{mg} / \mathrm{kg}=\mathrm{m}$ GAD $450 \mathrm{mg} / \mathrm{kg}+\mathrm{BBR} 50 \mathrm{mg} / \mathrm{kg}$ - BBR $50 \mathrm{mg} / \mathrm{kg}$ BBR $50 \mathrm{mg} / \mathrm{kg}+\mathrm{GAD} 450 \mathrm{mg} / \mathrm{kg}$

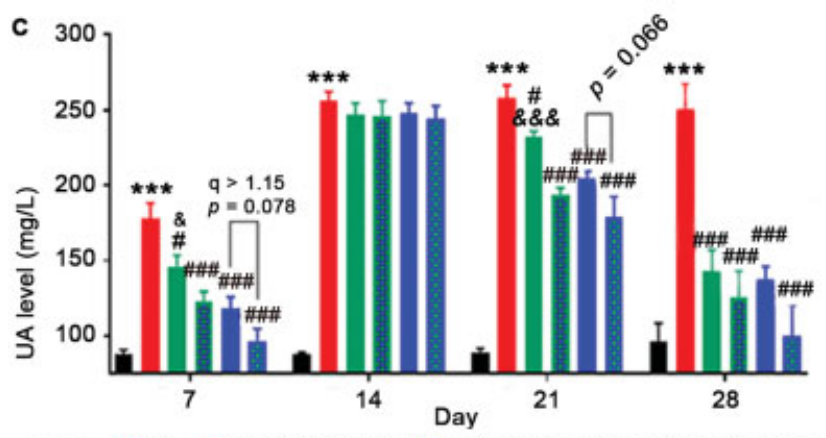

- $\mathrm{NC}=\mathrm{HN}=\mathrm{EAD} 450 \mathrm{mg} / \mathrm{kg}=\mathrm{EAD} 450 \mathrm{mg} / \mathrm{kg}+\mathrm{BBR} 50 \mathrm{mg} / \mathrm{kg}$ = BBR $50 \mathrm{mg} / \mathrm{kg}=$ BBR $50 \mathrm{mg} / \mathrm{kg}+\mathrm{GAD} 450 \mathrm{mg} / \mathrm{kg}$

Fig. 2 The combination use of GAD and BBR on (A) serum ALT, (B) $A S T$, and $(C)$ UA levels in rats with $H N$. Values were expressed as mean \pm SEM of eight animals. For statistical significance, one-way ANOVA was used followed by Dunnett's test. * $p<0.05$, versus NC group; ${ }^{* *} p<0.01$, versus NC group, ${ }^{* * *} p<0.001$, versus NC group. ${ }^{\#} p<0.05$, versus HN group; ${ }^{\# \# \#} p<0.001$, versus HN group. ${ }^{\&} p<0.05$, versus BBR $(50 \mathrm{mg} / \mathrm{kg})$ treated group; ${ }^{\& \&} p<0.01$, versus BBR $(50 \mathrm{mg} / \mathrm{kg})$ treated group, ${ }^{\& \& \&} p<0.001$, versus BBR $(50 \mathrm{mg} / \mathrm{kg})$ treated group. $q$, Jin method value $>1.15$, synergistic effect with BBR. ALT, alanine aminotransferase; AST, aspartate aminotransferase; BBR, benzbromarone; GAD, Gnaphalium affine D. Don extract; HN,

hyperuricemic nephropathy; NC, normal control; SEM, standard error of the mean; UA, urate.

hepatoprotection, the posttreatment of GAD (BBR + GAD) was more effective in urate lowering.

The synergistic enhancing effects were also validated by comparison of BBR alone at a dose of clinical usage (4.5 $\mathrm{mg} / \mathrm{kg} /$ day, divided in two doses) and BBR + GAD at half dose plus half dose $(2.25+225 \mathrm{mg} / \mathrm{kg} /$ day $)$ or half dose plus full dose $(2.25+450 \mathrm{mg} / \mathrm{kg} /$ day $)$. As shown in - Fig. 3, BBR $(2.25+2.25 \mathrm{mg} / \mathrm{kg} /$ day $)$ showed inhibition ratios of 40.8

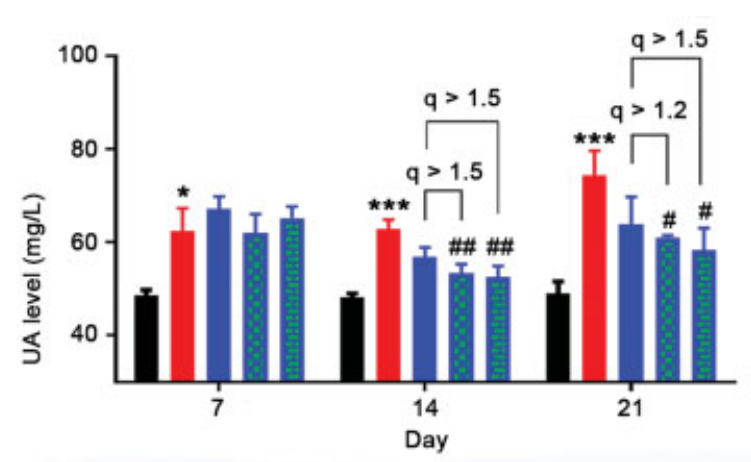

$\begin{array}{ll}=\mathrm{NC}=\mathrm{HN} & =\mathrm{BBR} 2.25 \mathrm{mg} / \mathrm{kg}+\mathrm{GAD} 225 \mathrm{mg} / \mathrm{kg} \\ \mathrm{BBR} 2.25+2.25 \mathrm{mg} / \mathrm{kg}=\mathrm{BBR} 2.25 \mathrm{mg} / \mathrm{kg}+\mathrm{GAD} 450 \mathrm{mg} / \mathrm{kg}\end{array}$

Fig. 3 Effects of BBR (4.5 mg/kg, twice daily at 1-hour intervals), BBR $(2.25 \mathrm{mg} / \mathrm{kg})+$ GAD $(225 \mathrm{mg} / \mathrm{kg})$, and BBR $(2.25 \mathrm{mg} / \mathrm{kg})+$ GAD $(450 \mathrm{mg} / \mathrm{kg})$ on serum UA levels in rats with HN. Values were expressed as mean \pm SEM of eight animals. For statistical significance, one-way ANOVA was used followed by Dunnett's test. * $p<0.05$, versus NC group; ${ }^{* * *} p<0.001$, versus NC group; ${ }^{*} p<0.05$, versus $H N$ group; ${ }^{\# \#} p<0.01$, versus HN group. $q$, Bürge method value $>1.2$ or 1.5, synergistic enhancing effect with BBR. BBR, benzbromarone; GAD, Gnaphalium affine D. Don extract; HN, hyperuricemic nephropathy; NC, normal control; SEM, standard error of the mean; UA, urate.

and $41.3 \%$ on day 14 and 21 , but there was no statistical significance in UA levels between BBR and HN group rats. The significant antihyperuricemic effects ( $p<0.01 \mathrm{vs.} \mathrm{HN} \mathrm{group}$ on day 14 and $p<0.05$ vs. HN group on day 21) of the cotreatments BBR + GAD were obtained with either $2.25+225$ or $2.25+450 \mathrm{mg} / \mathrm{kg} / \mathrm{day}$, respectively. By the Bürge method, $q$-values ( $>1$ ) indicating synergistic enhancing effects of cotreatments of two drugs were obtained and are displayed in - Fig. 3. The data demonstrated that combing a half-dose of BBR with half-dose (or full dose) of GAD was a "win-win"producing better hypouricemic activity and lower hepatotoxic risk.

\section{Discussion}

Hyperuricemia is a metabolic disorder associated with the development of pathological conditions such as gout. The suggestion that hyperuricemia is a risk factor for kidney diseases is supported by preclinical and clinical data. ${ }^{17}$ The urate-lowering therapy for decades or even lifetime is strongly recommended for hyperuricemia and gout patients. ${ }^{9,18}$ Unfortunately, the long-term medication of XOD inhibitors and uricosuric agents might be associated with a higher risk of nephrotoxicity and hepatotoxicity. ${ }^{18}$

A potential source of novel antihyperuricemic agents may be derived from medicinal herbs which also possess potential activities in protecting renal and hepatic function. Due to the therapeutic benefits for enhancing efficacy and reducing toxicity, monotherapy with herbs or combined treatment with chemical drugs would have great significance in preclinical or clinical study. G. affine proved to be a promise for the treatment of gouty arthritis and hyperuricemia, showing the ability to inhibit in vivo XOD activity, URAT1 expression, 
and anti-inflammatory activity in a PO-induced acute hyperuricemia mouse model and a MSU-induced acute gouty arthritis mouse model, respectively. ${ }^{6}$ Our previous study also confirmed that the ethanol extract (or fractions) of G. affine exhibited antihyperuricemic and nephroprotective effects in two acute hyperuricemia mouse models by hypoxanthine or UA induction, the butanol fraction activity was assigned to its uricosuric and nephroprotective action and the ethyl acetate fraction to its XOD inhibition. ${ }^{4}$ However, the two studies did not select the best validated experimental design for the efficacy assessment for two reasons: (1) time course of treatment: in animal models of these studies, treatment was initiated before the disease pathology is initiated (prophylactic treatment). This is in contrast to the clinical situation in which treatment is normally started after onset of symptoms and clear diagnosis. (2) Disease simulation: hyperuricemia is a chronic disease and is commonly associated with chronic kidney diseases. But acute animal models were applied in these two studies, the feature of hyperuricemia (long-term high UA levels and renal dysfunction) was not fully represented in the models. Thus, a chronic HN rat model $^{13}$ characterized by long-term hyperuricemia, severe glomerular sclerosis, renal interstitial fibrosis, renal dysfunction, and increased urine microalbumin excretion that better mimic the clinical condition was conducted in the present study.

This is the first study, to date, demonstrating that GAD inhibited the elevation of serum UA levels in chronic hyperuricemia status and attenuated the progression of dysfunction of HN rat kidney. Urate is known to be produced mainly in the liver where XOD catalyzes the oxidation of hypoxanthine and xanthine to it. The kidney excretes two-thirds of the UA produced daily. About $90 \%$ of the daily load of urate filtered by the kidneys is reabsorbed via specific anion transporters, including URAT1. Thus, the XOD enzyme and transporter URAT1 play crucial roles in hyperuricemia. ${ }^{19,20}$ In this longterm study (28-day HN induction and 26-day medication), GAD presented antihyperuricemic response at doses of 450 and $900 \mathrm{mg} / \mathrm{kg}$, with no significant difference from the positive control group (BBR $10 \mathrm{mg} / \mathrm{kg}$ treated group). GAD was able to inhibit not only the residual activity of liver XOD but the expression of kidney URAT1 in HN rats, and these results are in agreement with those in acute hyperuricemia mice. ${ }^{6}$ Additionally, it is also worth mentioning that GAD promoted nephroprotective activity at all tested doses, with $900 \mathrm{mg} / \mathrm{kg}$ more effective to reduce BUN and CRE production and relative kidney weight than BBR (the positive control), bringing their levels to normality. This is an obvious potential benefit of GAD, because hyperuricemia or gout patients are often seriously suffering from chronic kidney diseases.

To the best of our knowledge, it is the first report that the combination of both factors of HN condition and multiple high doses of BBR might potentiate the damage on the hepatic function in rats. Several studies also demonstrated that BBRinduced hepatotoxicity may be also associated with pathological conditions, including obesity ${ }^{12}$ and pre-existing liver injury. ${ }^{21}$

Notably, this is the first study reporting the beneficial effects of G. affine extract in co-treatment with BBR. Surpris- ingly, it appears that GAD has a dual effect on BBR: not only does it reduce BBR-induced hepatotoxicity, but it also potentiates the antihyperuricemic and renal protective activity of BBR. And posttreatment of GAD (1 hour later than BBR administration) promoted hepatoprotective and antihyperuricemic activities with more effectiveness of reducing ALT and AST production than its pretreatment ( 1 hour prior to BBR administration). Our data also indicated that reducing the dose of BBR and the addition of GAD could minimize the potential liver and renal dysfunction caused by BBR and chronic HN, while at the same time having sufficient therapeutic effects on the hyperuricemia.

Herbs can interact significantly with chemical drug treatments via cytochrome P450 (CYP450) isoforms. ${ }^{22,23}$ It is known that BBR is metabolized to 6- and 1'-hydroxy BBR by hepatic cytochrome P450 (CYP) 2C9 and CYP3A4, respectively. ${ }^{24} 1^{\prime}$-Hydroxy BBR is further metabolized to reactive intermediates and a series of glutathione adducts in human and mouse liver microsomes via formation of 1',6-dihydorxy BBR, which is responsible for BBR-induced hepatotoxicity. ${ }^{25}$ The previous phytochemical study showed that GAD is very rich in luteolin, apigenin, caffeic acid, and caffeoylquinic acids, which displayed potential inhibitory activity against CYP3A4 or 2 C9. ${ }^{26,27}$ Hence, the beneficial responses of GAD and BBR co-treatment might be due to the CYP3A- or 2Cmediated pharmacokinetic interaction via enhancing the bioavailability of BBR and reducing the exposure of its toxic metabolites.

In experiment 3, BBR $(2.25+2.25 \mathrm{mg} / \mathrm{kg})$ showed urate inhibition of $40.8 \%$ on day 14 and $41.3 \%$ on day 21 . In experiment 1, GAD showed urate inhibition of $36.0 \%$ on day 21 and $39.7 \%$ on day 27 at the dose of $450 \mathrm{mg} / \mathrm{kg}$. Thus, we speculated that BBR $(2.25+2.25 \mathrm{mg} / \mathrm{kg})$ and GAD $(450 \mathrm{mg} / \mathrm{kg}$ ) would be equally effective and applied the Bürge method in experiment 3 to discuss the synergistic enhancing effects of GAD and BBR co-treatment. Certainly, the GAD $(225+225 \mathrm{mg} / \mathrm{kg})$-treated group should be included in experiment 3 of future studies to directly compare the efficacy of GAD $(225+225 \mathrm{mg} / \mathrm{kg})$ and BBR $(2.25+2.25 \mathrm{mg} / \mathrm{kg})$.

\section{Conclusion}

All the study parameters show that GAD has antihyperuricemic, nephroprotective, and hepatoprotective effects and significantly improves the antihyperuricemic effect of BBR in HN rats. These effects may be attributed to both the pharmacodynamic and the pharmacokinetic herb-drug interactions, which should be validated in further studies. We suggest that co-treatment with GAD and BBR holds promise for the management of hyperuricemia and gout.

\section{Funding}

This work was supported by the National Natural Science Foundation of China (Grant No. 81603279) and the Natural Science Foundation of Shanghai (Grant No. 15ZR1440100). The authors would like to thank Mr. Tuyou Zheng and Mr. Genming Zheng for their help in herb collection. 


\section{Conflict of Interest}

The authors declare that they have no conflict of interest.

\section{References}

1 Chen D, Zhao J, Cong W. Chinese herbal medicines facilitate the control of chemotherapy-induced side effects in colorectal cancer: progress and perspective. Front Pharmacol 2018;9:1442

2 Wang W, Zhou H, Liu L. The role of Chinese herbal medicine in the management of adverse drug reactions of leflunomide in treating rheumatoid arthritis. Phytomedicine 2020;68:153136

3 Luo X, Ni X, Lin J, et al. The add-on effect of Chinese herbal medicine on COVID-19: a systematic review and meta-analysis. Phytomedicine 2021;85:153282

4 Liu F, Lin S, Wu C, et al. Hypouricemic activity of Gnaphalium affine D. Don [in Chinese]. Shenyang Yaoke Daxue Xuebao 2020; 37(01):12-20

5 Zhang W, Wu CZ, Fan SY. Chemical constituents from Gnaphalium affine and their xanthine oxidase inhibitory activity. Chin J Nat Med 2018;16(05):347-353

6 Zhang HJ, Li LN, Zhou J, et al. Effects of Gnaphalium affine D. Don on hyperuricemia and acute gouty arthritis. J Ethnopharmacol 2017;203:304-311

7 Li C, Wang E, Elshikh MS, et al. Extraction and purification of total flavonoids from Gnaphalium affine D. Don and their evaluation for free radicals' scavenging and oxidative damage inhabitation potential in mice liver. Arab J Chem 2021;14(03):103006

8 Jiang L, Piao J, Liu Y, et al. Protective effect of Gnaphalium tranzscelii against acute liver injury in mice [in Chinese]. Shizhen Guo Yi Guo Yao 2008;19(08):1901-1902

9 Chinese Society of Endocrinology CMA. Guideline for the diagnosis and management of hyperuricemia and gout in China (2019) [in Chinese]. Zhonghua Neifenmi Daixie Zazhi 2020;36(01):1-13

10 Lee MH, Graham GG, Williams KM, Day RO. A benefit-risk assessment of benzbromarone in the treatment of gout. Was its withdrawal from the market in the best interest of patients? Drug Saf 2008;31(08):643-665

11 Parzefall W, Schuppler J, Barthel G, Meyer-Rogge B, SchulteHermann R. Toxicological studies on a benzofurane derivative. I. A comparative study with phenobarbital on rat liver. Toxicol Appl Pharmacol 1990;106(03):482-499

12 Sun P, Zhu JJ, Wang T, et al. Benzbromarone aggravates hepatic steatosis in obese individuals. Biochim Biophys Acta Mol Basis Dis 2018;1864(6, Pt A):2067-2077
13 Liu N, Wang L, Yang T, et al. EGF receptor inhibition alleviates hyperuricemic nephropathy. J Am Soc Nephrol 2015;26(11):2716-2729

14 Liu X, Shang Q, Li C, et al. HPLC method for determination of uric acid in plasma of hyperuricemia model mice [in Chinese]. Yaowu Pingjia Yanjiu 2017;40(03):319-323

15 Dai T. Quantitative analysis in drug combination (author's transl) [in Chinese]. Zhongguo Yaolixue Tongbao 1998;14(05):479-480

16 Hoffmann D, Fuchs TC, Henzler T, et al. Evaluation of a urinary kidney biomarker panel in rat models of acute and subchronic nephrotoxicity. Toxicology 2010;277(1-3):49-58

17 Mende C. Management of chronic kidney disease: the relationship between serum uric acid and development of nephropathy. Adv Ther 2015;32(12):1177-1191

18 Strilchuk L, Fogacci F, Cicero AF. Safety and tolerability of available urate-lowering drugs: a critical review. Expert Opin Drug Saf 2019;18(04):261-271

19 Richette P, Bardin T. Gout. Lancet 2010;375(9711):318-328

20 Billiet L, Doaty S, Katz JD, Velasquez MT. Review of hyperuricemia as new marker for metabolic syndrome. ISRN Rheumatol 2014; 2014:852954

21 Takemura A, Gong S, Sekine S, Ito K. Inhibition of biliary network reconstruction by benzbromarone delays recovery from preexisting liver injury. Toxicology 2019;423:32-41

22 Zhang F, Huang J, Liu W, et al. Inhibition of drug-metabolizing enzymes by Qingfei Paidu decoction: implication of herb-drug interactions in COVID-19 pharmacotherapy. Food Chem Toxicol 2021;149:111998

23 Zhang F, Huang J, He RJ, et al. Herb-drug interaction between Styrax and warfarin: molecular basis and mechanism. Phytomedicine 2020;77:153287

24 Kobayashi K, Kajiwara E, Ishikawa M, Oka H, Chiba K. Identification of CYP isozymes involved in benzbromarone metabolism in human liver microsomes. Biopharm Drug Dispos 2012;33(08): 466-473

25 Yoshida M, Cho N, Akita H, Kobayashi K. Association of a reactive intermediate derived from 1 ',6-dihydroxy metabolite with benzbromarone-induced hepatotoxicity. J Biochem Mol Toxicol 2017; 31(10):e21946

26 Kimura Y, Ito H, Ohnishi R, Hatano T. Inhibitory effects of polyphenols on human cytochrome P450 3A4 and 2C9 activity. Food Chem Toxicol 2010;48(01):429-435

27 Rastogi H, Jana S. Evaluation of inhibitory effects of caffeic acid and quercetin on human liver cytochrome p450 activities. Phytother Res 2014;28(12):1873-1878 